42 | 2012

La communication, dimension oubliée de l'intelligence économique

\title{
La responsabilité de l'entreprise privée dans le culte de la minceur et dans la dérive pré- anoxerique de la femme active
}

\section{Francis Alain Guitton}

\section{QpenEdition}

\section{Journals}

Édition électronique

URL : http://journals.openedition.org/communicationorganisation/3981

DOI : 10.4000/communicationorganisation.3981

ISSN : 1775-3546

Éditeur

Presses universitaires de Bordeaux

Édition imprimée

Date de publication : 1 décembre 2012

Pagination : 227-244

ISBN : 978-2-86781-772-4

ISSN : $1168-5549$

\section{Référence électronique}

Francis Alain Guitton, «La responsabilité de l'entreprise privée dans le culte de la minceur et dans la dérive pré-anoxerique de la femme active ", Communication et organisation [En ligne], 42 | 2012, mis en ligne le 01 décembre 2014, consulté le 01 mai 2019. URL : http://journals.openedition.org/ communicationorganisation/3981; DOI : 10.4000/communicationorganisation.3981 


\title{
La responsabilité de l'entreprise privée dans le culte de la minceur et dans la derive pré-anoxerique de la femme active
}

\author{
Francis Alain Guitton'
}

La discrimination morphologique existe dans les entreprises du monde occidental. Les grands couturiers, les directeurs de casting du monde audio-visuel, les managers, les chefs de PME, les responsables des ressources humaines des grandes sociétés du CAC 40 savent de quelle manière la représentation physique peut être, pour une femme, prépondérante dans les secteurs de la relation humaine. Une femme projetée au contact d'éventuels acheteurs et consommateurs doit correspondre aux impératifs morphologiques acceptés par le plus grand nombre. La minceur est requise pour être présentée au public. Elle rentre dans les critères et les normes de beauté de l'époque et l'entreprise a su utiliser cet argument pour sublimer sa politique de croissance.

\section{Liminaire : quel est l'impact du physique sur la vie professionnelle ?}

Voyons ce que déclare un professeur d'économie fasciné par une science baptisée "pulchronomics » (ou économie de la beauté, le terme tirant son origine du latin "pulchritudo ", qui signifie beauté). Dans son dernier ouvrage $^{2}$, Daniel Hamermesh, enseignant à l'université du Texas à Austin, révèle, statistiques à l'appui, que les gens beaux ont des revenus plus élevés en moyenne que les autres. Ainsi un bel homme gagnera-t-il en moyenne $17 \%$ de plus qu'un type moche et une jolie femme, $13 \%$ de plus qu'une fille laide. Au cours de sa vie professionnelle, un bel employé recevra en moyenne 230000 dollars de plus qu'un travailleur jugé laid, une somme suffisamment rondelette pour inciter les gens appartenant à cette dernière catégorie à dénoncer l'injustice dont ils sont victimes. Le constat de cet économiste qui

1 Francis Alain Guitton est psychanalyste, psychologue, docteur en sciences de la communication et chercheur associé au laboratoire I3M Nice Sophia Antipolis. Écrivain il est auteur des Eclairs foudroyants, Albin Michel et d'Anorexie, les vrais coupables, France Europe Éditions, site internet www.francis-alain-guitton.fr

2 HAMERMESH D., Beauty Pays, Why Attractive People Are More Successful, (La beauté paie. Pourquoi les gens beaux ont plus de succès), Princeton University Press. 
base ses observations sur des études réalisées dans plusieurs pays? «La beauté est une denrée rare mais lucrative ». Une attachée de presse déclarait «Mon physique m'aide indéniablement dans mes relations avec mes clients, surtout masculins ; en général, ils acceptent très facilement de me voir, me présentent d'autres clients potentiels, me convient à des cocktails, le préjugé du "Sois coquette et tais-toi" a la peau dure ».

\section{Quel est l'impact de la minceur sur la vie professionnelle ?}

Si la beauté est un critère déterminant de réussite professionnelle, les critères de beauté actuelle restent des critères de minceur. Donc, dans une certaine mesure, la minceur est requise pour s'imposer dans les nombreux secteurs relationnels de l'entreprise et cette réalité détermine inconsciemment un positionnement discriminatoire dans les milieux professionnels. Le postulat de base semble être « soit mince et belle pour séduire et mieux vendre ou faire vendre ». Le contact avec le public et le consommateur sera plus facile et plus accessible pour une femme dont la représentation corporelle sera conforme aux normes anthropologiques de l'époque. Nous voulons, dans cet article, souligner les dangers d'une certaine dérive dans l'incitation à la minceur excessive. Celle-ci semble peu flagrante dans certains secteurs du monde du travail (industrie, production lourde, artisanat) mais semble dévastatrice dans les entreprises ayant un rapport étroit avec la représentation publique, la relation humaine, nous pensons au monde de la mode, de l'audio-visuel et de la publicité. Selon ces différents milieux ciblés, elle passera d'un degré notable et à peine insidieux à une connotation pathogénique, à l'extrême comme c'est le cas dans la haute couture.

Nous voulons dans un premier temps souligner les effets des mesures discriminatoires concernant les personnes de sexe féminin qui ne correspondent pas à ce moule stéréotypique de représentation corporelle dans le milieu professionnel. À ce propos le reportage d'un humoriste populaire sur une grande chaîne du service public nous pousse à la réflexion. Celui-ci avait fait intervenir une actrice au physique peu attrayant se trouvant près d'un feu rouge de Montparnasse demandant à un automobiliste de la conduire aux Champs Elysées. La réponse était systématiquement négative. À la même requête formulée par une jeune fille longiligne et jolie d'une agence de mannequin proche, les automobilistes réagissaient tous favorablement.

Cet exemple souligne bien sûr une évidence reconnue par tous : le physique d'une femme est un argument majeur de persuasion et la transposition sociale et professionnelle de cet atout est représentative dans tous les rouages de la société moderne. Nous constatons dans les milieux de la relation publique et du contact humain une utilisation manifeste de cette caractéristique élitiste alors que dans les milieux moins nobles (entretien, services, nettoyage), la représentation corporelle n'a pas d'importance et au contraire les personnes sont ciblées par défaut avec un physique difficile ou même ingrat. Y-aurait il 
dans ce domaine une forme de discrimination : certainement mais celle-ci est acceptée par la plupart et fait partie de nos mœurs. La question qui nous intéresse concernant les femmes en terrain psychologique fragilisé est de savoir si cet impératif de représentation corporelle façonné par les critères de minceur exacerbés qui sévissent dans notre société peut déterminer une dérive pathologique et anorexique.

La projection de l'image de la jeune femme sur un support public de grande audience semble être un facteur déterminant dans l'étiologie de la problématique. Le but de cet article n'est pas de parler de l'anorexie pathologique reposant sur des étiologies complexes et multifactorielles, mais de démontrer que certaines entreprises humaines ciblées ont une grande part de responsabilité dans la détermination d'un positionnement dangereux chez la femme active cherchant à s'insérer dans un milieu socioprofessionnel machiste. La minceur à tout prix semble être le message subliminal propagé par certaines entreprises puissantes et représentatives du système socio-économique occidental. La minceur excessive dans la représentation corporelle de la femme semble un fait de société, certes, mais l'étude des microcosmes actifs les plus impliqués dans ce phénomène social semble un champ d'investigation théorique intéressant et constituera la thématique de cette article.

\section{Les multinationales de la haute couture et de la mode, les grands couturiers imposant l'image des mannequins}

On a pensé qu'après l'adoption de lois condamnant l'incitation à la maigreur excessive dans de nombreux pays européens ${ }^{3}$, la situation allait sensiblement s'améliorer et que les mannequins sous-alimentés allaient être définitivement écartés des podiums. Il semble que les responsables des sociétés de haute couture ou de prêt-à porter et leurs DRH aient la dent dure et ne veulent pas remettre en question leurs critères de sélection. Faut-il attendre de nouveaux décès parmi les top-modèles pour, de nouveau, sensibiliser l'opinion publique et légiférer plus sévèrement ou plutôt pour appliquer la loi car la notion d'incitation à la maigreur semble floue et subjective et aucune sanction n'est encore tombée au niveau pénal.

Les grands couturiers ne se sentent ni concernés, ni répréhensibles du haut de leurs podiums parisiens ou étrangers. Au contraire, ils semblent conforter leur orientation dévastatrice comme l'atteste ce communiqué ${ }^{4}:$ "Plusieurs professionnels parisiens de la mode déplorent la minceur de plus en plus extrême exigée des mannequins mais jugent inutile toute mesure d'interdiction de défilés pour cause de maigreur. Les filles sont de plus en plus maigres et fines, la responsabilité

3 PROPOSITION DE LOI ( $\mathrm{n}^{\circ}$ 781) visant à combattre l'incitation à l'anorexie par Mme Valérie Boyer, Députée. Copie intégral du texte de la loi Boyer sur www.assemblee-nationale.fr/13/proposition.

4 Agence France Presse : Sous le titre «Mannequins : Les professionnels défendent les maigres » AFP 19.09.2006 - 9:29. 
de la minceur excessive de certains mannequins incombe aux créateurs et les agences essaient de respecter au mieux les créateurs, mais la mode est comme cela, elle a toujours été comme cela avec ces mannequins qui s'aspirent de l'intérieur».

"Globalement, maigreur reste synonyme d'élégance et ça n'est pas près de changer" déplore Sylvie Fabrégon, responsable de l'agence de mannequins Contrebande : "Les agences choisissent des filles très grandes, très fines, pour que l'habit tombe mieux et pour répondre aux demandes des stylistes et des annonceurs. Il est vrai qu'il n'y a pas de critères en termes de poids, mais il faut pouvoir rentrer dans un 34-36 et faire 1,75 mètre et plus. Les mannequins sont plus maigres quill y a vingt ans. Les plus pulpeuses ne sont admises que dans la mesure où "elles ont quelque chose en plus que le physique", comme Laetitia Casta, qu' "on n'emmerde pas" en lui disant: "maigris" ".

Ce témoignage met bien en évidence le scandale qui subsiste dans le milieu de la haute couture. On note que c'est la notoriété et la célébrité qui permet à une jeune femme connue pour ses formes plus arrondies (Laetitia Casta) d'avoir été sélectionnée longtemps et d'échapper au jugement redoutable des recruteurs bornés. Un top-modèle réputé pour son extrême maigreur connaît en revanche un regain d'intérêt spectaculaire ces derniers temps, malgré ses problèmes avec la justice pour ses addictions. Kate Moos est de nouveau de tous les défilés et les grands couturiers de la planète se l'arrachent. Rappelons, en matière de représentation du corps féminin, les conséquences désastreuses et dramatiques de cette appréciation généralisée dans tout un milieu qui impose la maigreur dans les défilés et écarte des sélections la moindre surcharge pondérale. Comment expliquer que les professionnels de la mode n'aient pas été sensibilisés par la gravité du phénomène et ne soient pas intervenus au départ, pour éviter des écueils insupportables pour la majorité des observateurs? Nous ne disons pas que tous les mannequins présentent cette morphologie malingre et sous-alimentée mais nous accusons ce milieu d'avoir permis à quelques-uns d'entres eux correspondant à cet aspect pathologique d'avoir été promotionnés sans réaction humaine ou sanitaire. Faut-il que les professionnels de la mode, les grands couturiers et les directeurs de casting aient été complètement aveuglés par leur subjectivité tendancieuse, pour que ce phénomène leur échappe et qu'ils soient restés inactifs et inopérants face à cette problématique douloureuse ? Comment expliquer qu'ils ont accepté des jeunes filles anorexiques sur leur podiums sans alerter les autorités de santé ou œuvrer à une législation pour combattre le phénomène sournois?

La question reste toujours posée. Il a fallu plusieurs décès de jeunes mannequins (voir le cas d' Ana Carolina Reston mannequin brésilien ${ }^{5}$ ) pour

5 Un mannequin de 18 ans meurt d'anorexie "Ana Carolina Reston avait été hospitalisée avec une infection urinaire qui s'est transformée en insuffisance rénale puis en infection généralisée. Elle ne pesait que 40 kilos, pour $1 \mathrm{~m} 74$. Le mannequin brésilien de 18 ans, Ana Carolina Reston, est morte d'anorexie à Sao Paulo, à la veille de son départ à Paris où elle devait poser pour des photos de mode " rapporte mercredi la presse locale. Mannequin depuis l'âge de 13 ans, Ana Carolina dernièrement ne s'alimentait que de pommes et de tomates. 
que l'opinion publique et non pas ce même milieu, scandaleusement non concerné et non responsabilisé par le problème, ne réagisse. N'oublions pas non plus ce qui constitue l'essentiel de notre réflexion dans cet article : l'impact iconographique de ces jeunes femmes mannequins sur un terrain constitué d'adolescentes particulièrement malléables et influençables qui arrivent sur le monde du travail et qui sont intéressées par la représentation médiatique de leur corps. La responsabilité des entreprises et multinationales du monde de la mode semble manifeste.

Les maitres du monde de la mode et les grands couturiers semblent avoir les clefs en main pour changer les données et il serait facile de lancer un processus de métamorphose progressive de la femme présentée dans leurs défilés. Faire davantage se rapprocher la représentation des corps féminins de la libido masculine semblerait facile et logique. Mais cela ne se fait pas. Les mannequins modernes n'ont rien de commun avec les Vénus du néolithique, ni avec les Aphrodites grecques, encore moins avec les modèles de Rubens ou de Jean Baptiste d'Ingres. Ils s'écartent même dans leur maigreur excessive des représentations androgynes des Egyptiens ou des périodes de libération de la femme au cours des sixties. Comment expliquer cette pseudo-dictature de la minceur chez les couturiers ? Qu'est-ce qui détermine le choix de la femme androgyne et l'effacement des formes féminines se rapportant à la maternité dès qu'il s'agit de représentation publique ?

Si nous nous plaçons d'un point de vue anthropologique et éthologique, le choix des partenaires du mâle était influencé par l'instinct de survie ; de ce fait, la maigreur était rédhibitoire. Le positionnement des couturiers et des responsables de casting se détache donc de ces impératifs libidinaux de base concernant la femme et les formes féminines marquées ; logiquement la symbolique et l'incarnation de la mère sont proscrites des défilés et rares sur les plateaux télé. Les couturiers ou directeurs de casting de ces entreprises coupables refusent d'imposer aux autres la représentation féminine libidinale de base pourtant prisée et acceptée par beaucoup d'hommes.

$\mathrm{Ce}$ qu'ils proposent s'oppose aux désirs des hommes en général. La stabilité du choix de leurs icônes, depuis des décennies, avec une constance imperturbable, montre la puissance du leadership qu'ils imposent. Les grandes entreprises programment la mode et les grandes lignes du prêt-à-porter féminin par le biais des créateurs de la haute couture ; ceux-ci imposent un dictat intolérable qui n'est contesté par personne. La puissance de ces multinationales absolutistes imposant le « bon goût » de leur "fashion system » est sans contre pouvoir et sans limites. Le fait de refuser inconsciemment de fournir aux hommes ce qu'ils attendent généralement des femmes en matière de critères esthétiques et de séduction constitue leur espace de liberté ciblé et tendancieux et les puissants de la haute couture et de la mode continuent à le défendre d'une manière acharnée. 
Sommes-nous en 2012 en train de constater un changement des méthodes pratiquées par les professionnels de ces multinationales de la mode?

Quelques articles de journaux féminins récents pourraient nous déterminer à penser cela. Le magazine Elle déclarait récemment ${ }^{6}$ : "Tara Lynn rompt les codes. Alors qu'elle s'était lancée dans le mannequinat pour arrondir ses fins de mois, l'Américaine de 27 ans est sollicitée de toute part. En effet, Tara Lynn n'a rien d'un top model tel quion le conçoit habituellement. Ses mensurations sont de 110×87×117. Elle mesure 1,76 mètre, pèse 85 kilos et s'babille en 48 ; elle est actuellement l'un des mannequins les plus côtés de la célèbre agence Ford Models. Le monde de la mode serait-il à un tournant?".

Le 2 mai 2010 on pouvait entendre sur une grande chaîne de télévision privée les paroles suivantes : "Il est toujours bon de voir une femme ronde qui s'assume, et surtout débordante de naturel. Tara a un visage poupon, un regard doux et un sourire franc. "Les femmes s'identifient peut-être plus facilement à moi", suppose-t-elle en simplement devant les caméras de "Sept à Huit ». Une réalité encore plus flagrante aux États-Unis, où $41 \%$ des Américaines font plus que la taille 14 (soit un 44 français environ). En France, la moyenne se situe entre le 40 et le 42 , et seules 5 femmes sur 100 entrent dans du $36^{7}$ ».

\section{Mourir pour son job en maigrissant !!}

Un autre mannequin grande taille, Crystal Renn qu'on a vu défiler effectivement chez Jean-Paul Gautier, Mango, Dolce \& Gabanna etc. a maintenant 23 ans et fait une taille 12 ce qui correspond au 42 des normes françaises. Mais ce mannequin a cependant sorti un livre assez troublant où elle raconte ses expériences dans le monde de la mode. Le titre est d'ailleurs très révélateur : Hungry (faim) est une chronique de son voyage fascinant dans le monde de la haute couture. Nous apprenons dans son livre sorti en septembre 2009 qu'avant d'avoir du succès, Crystal est passée par le mannequinat classique dès l'âge de 14 ans et on lui a demandé à cette époque suite à ses courbes rédhibitoires de perdre $23 \mathrm{~kg}$. Elle déclare dans son livre :

"À 17 ans, mon corps c'est complètement révolté. Je ne pouvais pas perdre plus de poids et j'ai réalisé que j'allais mourir pour un job ».

Quelle opinion peut-on avoir sur la problématique de la représentation du corps féminin dans le milieu de la mode à ce jour ? Le journal Le Monde déclarait le 29 septembre 2010 sous le titre : Les modèles sont-ils toujours soumis au diktat de la maigreur imposé par les entreprises de mannequinat? L'idée de bannir les mannequins trop maigres des podiums des défilés a fait long feu.

Elles sont toujours à la une des magazines et en tête des défilés pendant les Fashion Weeks. Pourtant, certains pays avaient évoqué il y a quelques années l'idée de ne plus engager de filles trop maigres. Alors que s'ouvre la semaine des défilés de Paris, après New York, Londres et Milan, le débat sur

6 Elle du 24 mars 2010 "Spécial rondes ».

7 Emission TF1 « Sept à Huit » 2 mai 2010. 
la maigreur des mannequins refait surface. Problème : tout le monde triche. Les mannequins et les agences ont trouvé la parade pour contourner les règles imposées en Espagne par exemple.

"L'agence vous met un soutien-gorge en plomb, avec une ceinture en plomb. Et on va se peser à l'examen médical ", explique Louise, mannequin depuis huit ans, pour qui ces mesures sont en fait une " grande blague». Les mannequins répondent à la demande des créateurs. Certains aiment faire défiler des filles très maigres, comme les maisons Balenciaga, Versace ou encore le couturier Karl Lagerfeld. "Personne ne veut voir des femmes avec des formes ", avait déclaré l'an dernier le créateur allemand. Qui est donc cet individu qui, voulant sans doute se mettre à la place des millions d'hommes médiatisés des pays occidentaux et n'étant pas du tout conscient de l'impact dévastateur que peuvent provoquer de telles paroles sur la structure psychique de milliers de jeunes filles adolescentes influençables, a l'aplomb d'affirmer une telle ineptie alors que nous venons de démontrer le contraire dans cet article?

Nous répondons « tout le monde veut voir des femmes avec des formes ». Il n'y a que le microcosme limité que ce couturier incarne qui affirme le contraire et les autorités de santé se doivent de réguler ce point de vue dangereux. À l'inverse, le Français Jean-Paul Gaultier est connu pour apprécier les filles plus en chair. Il a notamment fait de Crystal Renn, un mannequin grande taille, son égérie. Une directrice d'agence témoigne sur Europe 1: "J'ai un avis qui est absolument contraire à celui de tous les gens de la mode. On leur donne des filles magnifiques, minces et souvent ils me les rendent anorexiques pour rentrer dans leurs robes qui font du zéro, qui ne riment à rien et que personne ne pourra jamais porter ", dit-elle sous couvert d'anonymat (Source Europe 1) La partie suivante analysera les pôles d'influence des agences de publicité audio-visuelle des sociétés de diffusion de feuilletons-télé fleuves (soap, sitcoms ou novellas) qui inondent le marché des teenagers, des organes de diffusion de journaux (presse people) et de l'affichage urbain, des sociétés de productions cinématographiques.

\section{Les agences de publicité audio-visuelle}

"Donnez-moi un enfant de n'importe quel milieu et dites-moi ce que vous voulez que je fasse de lui : un scientifique, un artisan, un politicien ou même un criminel. J'atteindrai cet objectif». Voilà ce que pensait, en 1932, le psychologue américain Edward Chace Tolman'. Ce psychologue était convaincu que la publicité jouait un rôle manipulateur sur les adolescents. Ce qui nous intéresse dans notre étude est de positionner le corps féminin dans l'appréciation de l'adolescente ou la préadolescente. Aussi est-il utile d'analyser le rôle du corps féminin dans la publicité. Le corps de la femme a très tôt été utilisé pour faire

8 Le Monde, mercredi 29 septembre, 2010, 08:57.

9 TOLMAN, E. C. (1951), Behavior and psychological man: essays in motivation and learning, Berkeley, Univ. of California Press, Retrieved from «http://en.wikipedia.org/wiki/Edward_C._Tolman ». 
vendre et pour inciter à l'acte d'achat. Depuis le début de l'ère télévisuelle, le fait de présenter une jolie femme et d'utiliser les atouts fournis par son corps pour vanter les mérites d'une marchandise, a été exploité par toutes les entreprises se positionnant dans les biens de consommation et le service à la personne. Comme dans le cinéma et les feuilletons télévisés, les personnages utilisés dans les scenarii publicitaires ont reflété rigoureusement les critères de beauté contextuels. La publicité ne fait pas exception et va donc mettre en scène des femmes minces, d'une manière pratiquement absolue. Comme pour les feuilletons à passage régulier, nous mettons l'accent ici sur le côté « addict », particulièrement sensible chez les adolescentes et les préadolescentes, qui va constituer un danger potentiel dans l'élaboration d'une trame génésique de la maladie anorexique. La publicité martèle, passe quotidiennement et par ses stimuli visuelles et auditifs, marque progressivement le « disque dur » de notre structure psychique. Limpact cognitif de la publicité télévisuelle est d'une efficacité redoutable pour inciter à l'achat. De nombreuses études scientifiques le montrent. On peut estimer, par la vigueur de cet impact, le pouvoir qu'elle détient dans l'élaboration iconographique d'une image de corps féminin, dans l'esprit d'une jeune femme fortement influençable.

\section{Les organes de diffusion de journaux (presse people) et l'affichage urbain}

La publicité est présente aussi dans les hebdomadaires et l'affichage urbain. Si la lecture se perd chez les adolescentes et qu'il est de plus en plus rare de rencontrer des lectrices de romans ou d'essais chez les 12-18 ans, il existe un phénomène résurgent, ces vingt dernières années : l'engouement des jeunes adolescentes pour la presse people. En 1993, Prisma Presse lance Gala, une version haut-de-gamme de Voici. Dix ans plus tard, Lagardère arrive sur le créneau avec Public, suivi en 2005 par Closer. Ce dernier connaît un succès foudroyant, frôlant la barre des 400000 exemplaires dès sa première année de diffusion. Aujourd'hui, avec seulement cinq titres, les magazines people sont la $4^{\mathrm{e}}$ famille de magazines la plus vendue, avec 131 millions d'exemplaires diffusés en 2006. La presse people affiche une santé insolente, avec une diffusion en hausse de 3,34\%. Voici en reste le leader, avec 460000 exemplaires vendus en moyenne chaque semaine. Les « célébrités » issues de la téléréalité, puis du monde politique, ont notamment contribué à renouveler l'intérêt des lecteurs. Il semble donc évident que parmi les lectrices de cette nouvelle forme de presse figure une proportion importante de nos 12-18 ans et il est aussi évident que le phénomène de groupie concerne en priorité cette catégorie.

Il y a donc une clientèle de prédilection qui va alimenter ses fantasmes sur des vies, des activités, des moments de vacances, des relations amoureuses de personnages célèbres et subir, là aussi, de manière insidieuse, l'influence iconographique des corps publiquement dévoilés dans leur intimité, de ces stars adulées et admirées. L'affichage urbain a certainement et pour les mêmes raisons, un rôle à jouer sur la propagation de l'icône minceur parmi 
les jeunes filles adolescentes. On sait maintenant que la majorité des photos sont maquillées et ne correspondent pas à la réalité. On peut donc supposer que les professionnels fortement influencés par les impératifs de l'époque, interviennent très rapidement pour gommer une rondeur jugée choquante ou un amas de cellulite disgracieux, ce qui contribue à développer l'idée d'une généralisation de la norme promotionnée. La réflexion « elles sont toutes très minces et moi et moi et moi " prend une connotation très invalidante, développant même le complexe par comparaison, chez cette même jeune fille malléable. Cet aspect des choses n'est donc pas bénin et a certainement plus d'influence qu'on ne le pense.

\section{Les sociétés de diffusion de feuilletons-télé fleuves (soap, sitcoms ou novellas) qui inondent le marché des teenagers}

À partir des années soixante, la télévision s'est implantée progressivement dans les foyers. Peu à peu tous les membres de la famille furent exposés aux influences du petit écran. Les enfants et les adolescents se l'approprièrent très vite, sélectionnant d'emblée les programmes les concernant le plus. Parmi ces programmes figuraient les films venant de l'industrie du cinéma et par ce biais mettaient en branle les mécanismes psychologiques décrits ci-dessus, mais d'autres formes de programmes allaient considérablement influencer les non-actifs : les plus jeunes, les préadolescents et les adolescents après les cours, la femme au foyer et les personnes âgées (cette dernière catégorie étant cependant moins concernée par le phénomène que nous analysons). Ces deux produits télévisuels très influents furent les feuilletons à programmation régulière et la publicité qui s’installa dans la quotidienneté des familles.

\section{Les feuilletons journaliers et hebdomadaires}

Qu'un feuilleton programmé régulièrement et regardé avec assiduité et passion puisse déterminer une addiction astreignante n'étonnera pas la majorité des psychologues. Cette addiction est d'autant plus sévère qu'elle peut survenir sur des sujets jeunes et donc influençables et malléables. On a vu des adolescents entrer littéralement dans la peau d'un personnage qui sera sublimé à l'extrême tant la proximité intime est possible au travers des scénarios. Ce rapport étroit et régulier avec le héros ou l'héroïne d'une fiction peut même déterminer un déséquilibre affectif lié au fait que le clivage entre le réel et le virtuel est encore flou chez les plus jeunes.

Le romantisme d'une situation peut aussi déterminer une complicité sentimentale envahissante. En quoi cet état de fait bien connu et déjà observé et décrit par les scientifiques pourrait avoir un rapport avec notre thématique de recherche?

Il a bien entendu un rapport au niveau de la préemption à l'iconographie tel que nous l'avons décrit jusqu'à présent. La préadolescente ou l'adolescente qui rentre dans son processus progressif et largement inconscient d'addiction 
par rapport au personnage adulé du feuilleton va poursuivre avec lui son apprentissage par imitation et le mimétisme tout azimut sera lancé indépendamment de la volonté du sujet.

Si le personnage adulé prend les traits d'une femme d'une minceur excessive parce qu'elle correspond aux critères de beauté que nous avons définis plus haut et courants à notre époque parmi les actrices, il est préoccupant de se pencher sur cet état de fait imposé par une télévision de plus en plus omniprésente dans les familles.

Voyons ce que peut donner le phénomène dans les cas extrêmes. Marion est anorexique. En consultation lors de son anamnèse, elle déclara courant 2008 avoir été toute son enfance influencée par deux héroïnes de feuilletons américains qu'elle regardait régulièrement chaque semaine chez ses parents. Elle rêvait d'elles la nuit, copiait leur façon de s'habiller, de marcher et de s'exprimer. Ces deux héroïnes sont Ally Mac Beal, avocate new yorkaise brillante interprétée par Calista Flockhart, actrice américaine, et Sarah Michelle Gellar, héroïne de la série mondialement distribuée de "Buffy et les vampires ». En ce qui concerne ce cas clinique réel, on peut noter que, si le premier exemple concerne une actrice hollywoodienne connue pour sa pathologie anorexique diagnostiquée, il n'en est pas de même pour le deuxième exemple où l'actrice américaine est simplement mince et athlétique. Le cas clinique cité n'a pas pour but d'établir une relation de cause à effet direct puisque nous soulignons les étiologies multifactorielles de cette pathologie anorexique mais il a simplement une valeur indicative correspondant à une réalité rapportée par une jeune fille de vingt ans en souffrance. Hollywood qui fabrique d'ailleurs des centaines de «soap» pour adolescents et qui diffuse ceux-ci sur la planète, influençant des millions de jeunes filles de tous les pays industrialisés, est devenu une fabrique de starlettes toutes plus ou moins anorexiques. À l'image de Taylor Momsen, héroïne de « Gossip girl », dont la silhouette fond à vue d'œil et qui propose ses joues creusées, ses omoplates saillantes et ses bras fil de fer régulièrement aux teenagers admiratifs. Le phénomène est récent et s'amplifie ces dernières années (2008-2009). Il y a pléthore de ce genre de feuilleton (Degrassi nouvelle génération, 90210, Cw etc.) pour ados où les jeunes actrices sont triées sur le volet pour leur IMC (limite $: 1,73 / 46 \mathrm{~kg} ; 1,60 / 40 \mathrm{~kg}$ ) pour les « lolicéennes » mises en scène ${ }^{10}$.

\section{Les sociétés de productions cinématographiques}

Les modèles féminins reconnus et imposés par l'industrie du cinéma vont établir des canons de beauté et d'esthétisme indéboulonnables, qui vont prendre place dans l'inconscient collectif, influençant les attitudes et le

10 La jeunesse dorée new-yorkaise a le vent en poupe. Après une série dérivée qui devrait voir le jour à l'automne, la série à succès Gossip Girl a donné des idées à la chaîne américaine Bravo. La chaîne câblée américaine va en effet produire une version télé-réalité du feuilleton pour ados. Le principe est simple : la chaîne va planter ses caméras du côté des lycées privés de Manhattan et suivre les péripéties de jeunes étudiants privilégiés. 
comportement des habitants des nations industrialisées face à la séduction, l'alimentation et l'acceptation de soi. Ce phénomène semble redoutable chez la préadolescente et l'adolescente. La relation humaine et le contact entre les hommes et les femmes dans leurs activités quotidiennes vont en être fortement influencés. C'est pour cette raison que nous parlons de l'élaboration d'un monde de l'apparence et de ses icônes absolutistes. Or, le danger vient du fait que l'icône minceur semble s'imposer dans tous les rouages médiatiques d'une manière autoritaire. Examinons un de ces rouages en évaluant l'impact qu'il a respectivement sur l'acceptation et l'adoption d'une apparence et d'une représentation du corps féminin spécifique : l'apparence minceur.

Le média susceptible de créer des idéaux féminins, se positionnant comme une machine à rêves et développant des images féminines iconographiques par excellence, fut bien le cinéma qui augmenta considérablement son influence sur les masses populaires dès l'après-guerre. Les studios hollywoodiens créèrent des personnages féminins adulées, désirées et sublimées comme jamais cela ne s'était observé dans l'histoire du monde. La tenue vestimentaire, le choix des prénoms, le cadre de vie, les goûts, les aspirations de chacun et bien sûr les critères de beauté féminine furent conditionnés par les vedettes du grand écran. On se mit à s'habiller, à se maquiller, se déplacer, à s'entourer d'objets similaires, de manière à ressembler le plus possible à l'actrice en vogue. Le mimétisme cinématographique était né. C'est ainsi que des personnages tels que Greta Garbo, Marlène Dietrich, Jeane Harlow, Bette Davis, Vivian Leigh, Rita Hayworth, Michèle Morgan, Danielle Darrieux et plus tard Silvana Mangano, Liz Taylor, Sophia Loren, Anita Ekberg et Gina Lollobrigida influencèrent considérablement, avec leurs formes en général pulpeuses, les générations d'avant et d'après guerre dans les nations occidentales. Les années soixante et suivantes imposèrent leurs icônes plus longilignes liées, comme nous l'avons vu, à l'androgynie consécutive à la libération de la femme. De Jean Seberg dans la nouvelle vague introduite par Goddard et son « À bout de souffle » en passant par Hepburn, Lana Turner, Grace Kelly, Jane Birkin ou Jane Fonda la mode était cette fois du côté de la femme féline, sportive, plus garçonne, et aux formes plus estompées.

Les années qui suivirent développèrent un certain éclectisme mais le culte de la minceur s'imposa progressivement dans les années 80 et commença à influencer fortement le cinéma et la sélection des actrices par les metteurs en scène. Actuellement les actrices stars ou les actrices en vogue sont dans une grande majorité très minces, certaines à la limite de la maigreur. Angelina Jolie, Nicole Kidmann, Keira Knighley sont quelques-unes des comédiennes les mieux payées d'Hollywood de 2000 à 2012 et elles se caractérisent par une morphologie très longiligne, à la limite de l'anorexie pathologique pour cette dernière. 


\section{Le point de vue anthropologique}

Le stéréotype en matière d'apparence du corps féminin s'est imposé dès le début de l'histoire et cette tendance s'est poursuivie jusqu'à nos jours, autoentretenue dans l'ordre par les catalyseurs politiques, religieux, médiatiques et maintenant, comme nous l'avons vu ci-dessus, par l'entreprise amorçant et amplifiant le processus. Ainsi de la Vénus de Willendorf à Kate Moos ou à une autre star malingre de notre époque, les images sacrées sont pléthores. Tous ces personnages adulés pour leur corps se sont successivement imposés comme icônes sacrées pour les générations d'hommes qui ont vécu sur la terre ces dix derniers millénaires. Ce qui change et qui pose problème à la jeune femme active du XXI siècle, ce sont ces icônes modernes imposées par les médias et la mode. Marie-Joseph Bertini parle dans son livre des différentes figures imposées par les hommes pour la femme au cours des siècles ${ }^{11}$.

Parmi ces figures, nous trouvons la muse. La muse est une création masculine pour étayer sa propension iconique. La nécessité que l'homme s'est imposée de sublimer des figures sacrées en matière de représentations féminines l'a déterminé à positionner ce rôle féminin passif d'inspiratrice mystique à l'origine de sa créativité artistique. "La différence entre la figure de l'égérie est celle de la muse est cependant très subtile. L'égérie est inspiratrice, la muse, elle, est inspiration. La muse est la figure emblématique d'une passivité ontologique, une sorte de statufication de la femme figée dans le glacis de son être. La figure de la muse à la différence de celle de l'égérie implique un renoncement plus marqué une dimension sacrificielle inhérente à l'interdiction faite aux femmes de se penser non plus seulement comme actrice mais encore comme créatrice».

Nous constatons que si l'homme a exploité sa supériorité physique et sexuelle pour imposer ses normes phalliques et libidinales à la femme, il a parallèlement et indirectement imposé le statut de muse à sa partenaire, l'installant dans une passivité confortable au cours des siècles passés. Celle-ci a d'ailleurs accepté avec docilité, complaisance et jouissance, ce statut qui valorisait inconsciemment son instinct de séduction et son narcissisme identitaire, en minimisant sa part active dans sa représentation sociale au sein de l'entreprise moderne. C'est ainsi qu'au cours des siècles la femme a accepté qu'on lui détermine ses apparences physiques et la représentation de son corps est toujours passée au travers du filtre modulateur du phallocrate absolutiste. C'est cette tendance qui sévit toujours, à l'intérieur des structures socio-économiques et associatives, dans l'esprit des recruteurs, DRH et chefs de personnel hommes, qui déterminent leur choix et leur sélection inconsciemment. Bertini ajoute : "Le rôle des médias en la matière est d'autant plus ambigu, quills distillent un double message en même temps quils entretiennent, avec autant de constance que de régularité, l'idée du bien-fondé de l'invisibilité de la femme dans la sphère publique. Ils enjoignent à celles-ci de surexposer l'image de

11 BERTINI M.- J., Femmes, le pouvoir impossible, Édition Pauvert 2002 p. 31 
leur corps. Le corps dévoilé, offert, instrumentalisé des femmes est l'exacte contrepoint de la durabilité de leur absence politique. La surreprésentation du corps féminin manifeste de façon violente l'organisation de son éviction du corps politique. Là où l'imaginaire des hommes sature l'espace social de ses rappels obsessionnels au corps de la femme, c'est-à-dire à la femme comme corps, les femmes n'exercent qu'un pouvoir factice contrarié par de multiples obstacles dressés entre elles et le monde ».

Ce raisonnement est à tempérer cependant compte tenu de l'évolution certaine de la représentativité de la femme en entreprise. La femme a conjuré progressivement cette tendance séculaire et des progrès sensibles sont à noter depuis les années 1980 mais ce statut de muse passive influence, comme l'affirme Bertini, encore considérablement les décideurs du microcosme politique et surtout les instances dirigeantes des grandes sociétés, ce qui fait que la parité est loin d'être obtenue, dans le milieu politique comme dans les conseils d'administrations et les directoires.

Bertini ajoute : "Contre toute apparence, le même mouvement anime certains pays islamiques, pour ne citer qu'eux, ensevelissant le corps des femmes dans d'épaisses étoffes tout en leur déniant un quelconque accès à la vie publique. Alors, à tout prendre que vaut-il mieux? Un corps diabolisé ou bien un corps fétichisé? Un corps nié par le voile ou martyrisé par le "tchadri" afghan et tout ce qui l'accompagne, ou bien un corps appareillé par une érotisation urbaine, plaquée dans les esprits comme sur les abribus? Entre l'imam et le publicitaire, y a-t-il une si grande différence? Cet autre grand média qu'est la publicité constitue un dispositif culturel qui, précisément parce qu'il ne ressortit pas d'une contrainte directe et implacable comme le religieux, possède une redoutable efficacité. En prônant un rapport ludique et superficiel au réel, le publicitaire inscrit avec force, le spectateur dans le champ d'un devoir être et paraître qui s'adresse en priorité aux femmes. La publicité apparaît bien pour ce qui est vraiment: une catéchèse des temps modernes ${ }^{12} »$.

Nous compléterons la réflexion en ajoutant que la publicité est loin d'être la seule responsable de cet état de fait. Comme nous l'avons vu précédemment, tous les moyens mis en œuvre pour présenter le corps-icône de la femme dans sa représentation " minceur oblige " à notre époque sont concernés. Nous entendons en plus de la publicité, les plateaux de défilés de haute couture, les feuilletons télévisés et sitcoms de tout acabit, le cinéma et ses stars, la diffusion des journaux people, le show-business en général. Il est évident que le monde de l'entreprise ne peut qu'obtempérer et ses cadres supérieurs ne peuvent que reproduire la tendance séculaire.

La prise de pouvoir de la femme dans l'entreprise ne pourra s'obtenir que lorsque ces dernières barrières tomberont, lorsque la vision de l'homme recruteur aura été débarrassée de ces imprégnations cognitives stagnantes.

Il est intéressant de se pencher sur le point de vue d'une anthropologue ${ }^{13}$. Dans son introduction au symposium de Locha au Palais de la Découverte,

12 BERTINI M.- J., 2002, p. 34.

13 Annie Hubert est anthropologue, directeur de recherche au CNRS, UMR « Adaptabilité biologique et 
Annie Hubert, Directeur de recherche au CNRS, pose des questions fondamentales pour l'anthropologie. "La représentation du corps féminin se transforme lentement pour devenir celle d'un corps très mince, voire éthéré, jeune et performant, libéré de tout carcan. La moralisation du devoir de santé a entraîné une moralisation de l'esthétique. Et c'est très jeune que se forge l'idée que l'on a de son propre corps et de son apparence, que l'on se soumet aux normes exigées par le groupe ou quion tente parfois de les combattre pour affirmer son existence, que faire pour que nos enfants grandissent avec une image positive de leur corps. Sans oublier de rappeler qu'aucune norme, aucune influence, si contraignante soit-elle, n'intervient dans ces qualités bien plus importantes que sont le charme et l'ouverture vers les autres».

\section{Conclusion}

L'image du corps, la représentation du physique féminin a, sans aucun doute, un contenu stratégique qui permet d'éloigner la charge normative et la vision thérapeutique. Une présentation du corps d'une jeune fille lors d'un défilé de haute couture a le pouvoir de conquérir le regard des autres et d'incorporer un rôle, une identité sociale en suivant une règle de manière lisible, consensuelle et la résistance sanitaire s'effondre. La beauté reconnue socialement a une dimension symbolique, elle permet d'alléger le contrôle social, elle facilite la prise de rôle et la socialisation. Ces jeunes filles sous-alimentées sont capturées par leur identité sublimée. Jean-Claude Kaufmann dans son livre Corps de femmes, regard d'hommes montre comment la tolérance du regard plagiste est façonnée par des critères esthétiques ${ }^{14}$. L'acceptation du sein nu dans un endroit ciblé : la plage, passe par la reconnaissance de l'harmonie et de la satisfaction sensorielle du voyeur. Dans le cas contraire, il sanctionne. Seulement, dans le cas qui nous préoccupe, la beauté ne permet pas à ces femmes fragilisées de garder des marges de liberté pour éventuellement expérimenter, évoluer, changer d'orientation (à la limite de changer de position corporelle).

Ces jeunes femmes sont aliénées par leur image et perdent le contrôle sous la pression des enjeux (argent, gloire, luxe). La capacité de distanciation, de l'aisance analysée par Bourdieu, « cette sorte d'indifférence au regard objectivant des autres qui en neutralise les pouvoirs, suppose l'assurance que donne la certitude de pouvoir objectiver cette objectivation, s'approprier cette appropriation, d'être en mesure d'imposer les normes de l'aperception de son corps ${ }^{15} »$. La femme moderne face à l'entreprise perdrait-elle cette liberté, ce pouvoir dissociatif dans le milieu asservissant de la mode et de la représentation médiatique ? Il faut également souligner le fait que la mode est aussi un produit de consommation et la consommation érigée en valeur sociale et identitaire s'applique au corps

culturelle ». Spécialisée en anthropologie nutritionnelle et de la santé, elle a écrit et publié de nombreux ouvrages sur l'alimentation et la santé.

14 KAUFMANN J.-C., Corps de femmes, regards d'hommes, Paris, Pocket, p. 268.

15 BOURDIEU P., La distinction : critique sociale du jugement, Paris, Éditions de Minuit, 1979. 
de la femme et suppose une généralisation de la forme marchande de celui-ci qui devient forme universelle. Il est question de réification dans cet article et il renvoie tout particulièrement aux analyses de Georg Lukács concernant le fétichisme de la marchandise dans la société capitaliste. Lukács donne au terme de réification une connotation fétichiste constituant le concept d'aliénation. La dépossession du sujet et de l'objet, générateur de l'aliénation est une idée marquante développée par le philosophe allemand. Nous mettons bien en évidence le caractère aliénant du positionnement de la femme moderne face à ces multinationales puissantes imposant leur image stéreotypique du corps féminin ${ }^{16}$. Se pourrait-il que l'entreprise puisse établir des normes, des influences contraignantes en matière de présentation physique chez la femme, estompant les qualités professionnelles, humaines et la compétence objective de celle-ci ?

Se pourrait-il que les instances au pouvoir décisionnel des grandes entreprises aussi bien que des petites structures économiques puissent se laisser guider, d'une manière abusive, par ces critères physiques de minceur et de présentation subjective dans leur choix de recrutement au détriment de ce qu'appelle Madame Hubert le charme et l'ouverture sur les autres ? Notre article aura l'avantage d'avoir fourni une ébauche de réponse. Notre réflexion, qui souligne et pose l'hypothèse de la part de responsabilité du monde de l'entreprise et surtout l'entreprise axant son activité dans le monde de l'apparence, des médias et de la mode sur le phénomène de l'incitation à la minceur excessive, devrait donc faire réfléchir les personnes ayant pouvoir décisionnel dans tous les milieux concernés. Les représentations féminines mises en scène par la télévision, les feuilletons pour adolescentes, le cinéma, la mode, la haute couture, la presse et la publicité et le positionnement de certaines entreprises ayant des rapports avec ces milieux peuvent être une incitation ouverte à la maigreur excessive et cette incitation peut être néfaste pour la santé. Elles déterminent même des jugements réprobateurs et terriblement traumatisants pour les filles dès le plus jeune âge dans les cours d'école jusqu'à leur présentation sur le marché du travail. Que faut-il donc faire afin que les choses évoluent dans le sens d'un changement des icônes morphologiques modernes imposées à la jeunesse influencée par ces médias inamendables ? Les professionnels de la mode, de l'audio-visuel, responsables des castings ou autre recrutement sélectif, donnant accès aux métiers de la représentation ou de l'image, ne veulent pas entendre raison sur le règlement de ce phénomène de santé publique et veulent garder leurs critères de sélection tendancieux et dangereux. Comment les arrêter ? N'y-a-t-il pas que les démarches juridiques ponctuelles débouchant sur des procès très médiatisés

16 VINCENTI L., "Fichte, Lukacs, et la réification », conférence prononcée dans le cadre du séminaire « Marx au XXIe siècle » (C.H.S.P.M., Paris-I Sorbonne), journée d'études consacrée à la réification, de Lukacs à Honneth. Site du séminaire « Marx au XXI siècle » url : www.marxau21.fr. 
qui pourraient réellement inverser les tendances et faire cesser la pratique de « la maigritude » constatée dans ces milieux ciblés ?

$\mathrm{Ne}$ pourrait-on envisager que les instances décisionnelles des milieux professionnels dont nous avons, sans aucun doute, établi la part de culpabilité dans l'étiologie du phénomène social dévastateur, puissent payer d'une certaine manière et que les victimes de la discrimination inconsciente de ces enjeux commerciaux soient dédommagées sur le plan financier. Ce serait une perspective certainement dissuasive qui aurait l'avantage de permettre, dans les pays soumis à ces courants médiatiques insidieux, de combattre une image de la femme stéréotypique correspondant à une iconographie sociale dévalorisante qui n'a aucun rapport avec les compétences professionnelles et sociales de cette dernière. Alors faut-il davantage solliciter la loi Boyer du 8 avril 2008 et faire mordre la poussière à ces « Pratiquants de la Maigritude professionnelle » et établir des cas de jurisprudence salutaires pour stopper ce système tendancieux dangereux ? Nous le pensons sincèrement.

\section{BIBLIOGRAPHIE}

AGENCE FRANCE PRESSE, « Mannequins: Les professionnels défendent les maigres » AFP 19.09.2006 - 9:29

BERTINI M.- J., Femmes, le pouvoir impossible, Édition Pauvert 2002.

BOURDIEU P., La distinction : critique sociale du jugement, Paris, Éditions de Minuit, 1979.

BOYER V., Assemblée nationale constitution du 4 octobre 1958 treizième législature enregistré à la présidence de l'assemblée nationale le 9 avril 2008. Rapport fait au nom de la commission des affaires culturelles, familiales et sociales sur la proposition de loi ( $n^{\circ} 781$ ) visant a combattre l'incitation à l'anorexie par Mme Valérie Boyer, députée.

Elle du 24 mars 2010 « Spécial rondes ».

EMISSION « Sept à Huit », TF1, 2 mai 2010.

HAMERMESH D., Beauty Pays Why Attractive People Are More Successful, (La beauté paie. Pourquoi les gens beaux ont plus de succès), Princeton University Press.

KAUFMANN J.-C., Corps de femmes, regards d'hommes, Paris, Pocket 2010.

LE MONDE mercredi 29 septembre 2010, 08:57.

MAGAZINE «Elle » du 24 Mars 2010 « Spécial rondes ».

TOLMAN, E. C., Behavior and psychological man: essays in motivation and learning, Berkeley, Univ. of California Press, 1951, Retrieved « http://en.wikipedia.org/wiki/ Edward_C._Tolman ».

VINCENTI L., "Fichte, Lukacs, et la réification », conférence prononcée dans le cadre du séminaire " Marx au XXI siècle » (C.H.S.P.M., Paris-I Sorbonne), journée d'études consacrée à la réification, de Lukacs à Honneth. Site du séminaire « Marx au XXI ${ }^{e}$ siècle » url : www.marxau21.fr 
Résumé : La discrimination morphologique existe-t-elle, d'une manière exacerbée, dans le monde de l'entreprise ? Peut-on affirmer que, d'une manière tendancieuse, certaines sociétés, de la microstructure à la multinationale, puissent recruter plus facilement une femme belle et mince qu'une femme défavorisée sur le plan physique ? Cela paraît évident et peut même paraître logique pour certains, si l'activité demandée requiert un contact relationnel et public mais quelle peut être la limite décente et morale de cette réalité conceptuelle et sociale ? Quelles sont les dérives dangereuses d'un tel positionnement accepté par nos contemporains ? Notre objectif sera de sensibiliser le lecteur sur les conséquences d'une dérive sociétale dangereuse, sur la nécessité de réagir pour éviter l'écueil tendancieux et pathogène pour les sujets fragilisés et de préconiser une prévoyance sanitaire éclairée et équilibrée au sein des instances de recrutements de toutes structures professionnelles et associatives.

Mots-clés : Entreprise, décideurs, minceur, femme, mode, mannequin, corps, représentation physique, medias, apparence, haute couture.

Abstract : Does the morphological discrimination exist, in a aggravated way, in the business world? We can assert that, in a biased, certain way companies, of the microstructure to the multinational, can recruit more easily a beautiful and thin woman than a woman discriminated on the physical plan? It countered evident and can even be logic for some, if the wanted activity requires a relational and public contact but what can be the decent and moral limit of this abstract and social reality? What are the dangerous drift of such a positioning accepted by our contemporaries? We suggest, at first, making the analysis of the effect of the multinationals of the fashion design and the fashion and defining the influence of the fashion designers imposing the image of the models. Our objective will be to make sensitive the reader on the consequences of a dangerous societal drift, on the necessity of reacting to avoid the biased and pathogenic stumbling block for the weakened subjects and to recommend a sanitary foresight enlightened and balanced within the authorities of recruitments of any professional and associative structures.

Keywords : Company, Business, Decision-Makers, Thinness, Woman Fashion, Models, Body Physical Representation, Fashion Designer, Appearance. 
